\title{
Research to Promote Deep Learning in Learning Commons
}

\author{
Chun $\mathrm{Li}^{1, \mathrm{a}}$, Yingbin $\mathrm{Wei}^{2, \mathrm{~b}}$ \\ ${ }^{1}$ Library of Hainan University, Haikou, 570228, China; \\ ${ }^{2}$ Hainan College of Software Technology, Haikou, 571400, China. \\ achunli32895@163.com; byigch009@126.com;
}

Keywords: information commons, deep learning, model.

\begin{abstract}
This article clarifies the possibilities and advantages of deep learning which LC support to be realized, from the concept and connotation of the information commons, deep study, and propose the design principles and design models of LC promoting deep learning, specifically describes the point which must be noted in model design of promoting deep learning.
\end{abstract}

\section{Introduction}

Learning Commons (called LC) precisely meets the needs of higher education reform, which is gradually developed from Information Commons (called IC). It merges digital technology, modern equipment and a variety of information resources, gives all-round support to learning process of groups of learners through a variety of effective means, and cultivates students' information literacy ,such as self-learning ability, information retrieval capabilities, knowledge creation, innovation and practice etc. Deep learning is the basis, premise and guarantee for the development of innovative capacity, which involves more complex learning process compared to the shallow learning , and is an advanced study stage. How to use LC to promote deep learning has become a hot research topic multidisciplinary competing concerns.

\section{Outline on LC and Deep learning}

\subsection{From Information Sharing space to LC}

IC focused on provide learning spaces, reference service and technical support to readers, as well as electronic resources, multimedia, print resources and services integrated "one-stop "service in the early. With the reform and change of the educational way and the students learn way, the user has more expectations and higher demand for library services and IC. Among the many development directions, LC can be described as the most vitality, the fastest growing. Therefore, IC gradually developed into LC, LC can be considered a new stage of IC development.

Change from IC to LC, in addition to containing the original property and connotation of IC, focused on clearing and developing full support for the student collaborative learning in university library, reflecting support for group communication, collaboration and guidance. University Library LC has the following characteristics: Purpose; Dynamic; Equality and Sharing; Collaborative; Experience etc.

LC not only has the perfect hardware resources and abundant information resources, but also seamlessly naturally embeds hardware and software resources into the user's learning and teaching-research activities. LC is based on interdisciplinary and intersectional collaboration, combined information resources, technical resources, space resources, human resources in one set to build interactive learning environment, which includes three-dimensional structure of virtual, physical, support to promote mining tacit knowledge among staff, and to help users learn success and knowledge creation ${ }^{[1]}$.

\section{2 depth study}

The mid-1950s, Terence Matron and Roger Salvo in a joint publication proposed two concepts of study and shallow depth in "the essential difference between the results and the process of learning." In their read article experiments, it considered that passive learning belonged to shallow 
learning what students did in order to cross the border, thus separated knowledge, mainly memorized and recited the main, did not understand isolated learning; A higher level of thinking belongs depth study, which students can understand the basic content by the reading, seize the subject of the article, repeat them back through their own words, become a knowledge of their own mind, and can actively put the knowledge in the article contact with their professional, realize the knowledge application migration ${ }^{[2]}$.

The main criticism has five basic characteristics which were Critical Understand, Information integration, Problem Solving, Migration use and Construction reflection.

(1) Information integration

Information integration include integration of process and content. It requires learners to link the isolated content into some existing knowledge and experience by using different learning strategies, learning methods.

(2) Critical Understand

Depth of learning is based on understanding, which emphasize learner critically acquire new knowledge and ideas and put them into the existing cognitive structure, establish the multiple connection between the various viewpoints.

(3) Construction of Reflection

Construction of Reflection refers a process, which learners realize knowledge assimilation and adaptation through the new-old experience double-sided interaction on the basis of information integration, adjust the original cognitive structure, and construct the generated results by review, analysis and adjustment. Construction of Reflection can be said is the essential difference between shallow learning and Deep learning.

(4) Migration use

Deep learning requires the judgment and grasp to key elements, based on a deep understanding of the new situation, among them analysis is put on the complexity and diversity of the concept, thinking principles are restructured of the migration application.

(5) Problem Solving

Another important difference between shallow learning and depth study is that whether he can use knowledge to solve problems in new situations. Learning to solve complex, ill-structured problem is the expression of depth-learning and the only way ${ }^{[3]}$.

\section{Support Role LC to depth study}

Advances in technology, maturing network technology, integration of information technology and education, thus make learning resources more and more abundant, human has entered the era of digital learning. Learning is always in a certain time and space occurred, more and more people teach and learn through digital environment, where LC is such one. It has characteristics: diversification of information content display, Intelligent of knowledge processing, shortcut of information transmission and virtual learning environment et. LC uses individual wisdom, takes advantage of the collective wisdom to solve problems or complete a certain learning tasks and achieve spiral of knowledge and knowledge innovation, playing a good supporting role to depth of College Students through collaboration, exchange and sharing.

\subsection{Situational support}

In teaching or learning practice, it is unlikely to be achieved that teaching or learning fully implemented in the real world. The constructivist learning theory focus on creation of teaching real situation, which is the "meaning construction" premise. LC, as a one-stop service model and collaborative service environment designed specifically around integrated digital environment, with its technical advantages such as virtualization, intelligent, Macross et., can reduce temporal and geographical differences, helping students to produce a strong motivation and cognitive conflict, constituting a significant of teaching or learning content to students.

\subsection{Support Resources}

LC combines learning resources, information resources, technical resources and human resources, providing the actual situation of seamless interactive platform which can give users 
comprehensive stimulation from multiple senses, not only helps users access and maintain the knowledge, but also helps users to establish the contact of previous knowledge or experience and learned one.

\section{3 interaction support}

Learning is to initiate the construction of meaning by communicating and collaborating with others. Any kind of learning activities, have a social nature, "deep learning" is no exception. LC promotes cooperation and exchanges, and promotes knowledge sharing, can effectively support exploring, consultation, facilitate the development of various forms of conversation and collaboration among students, learning partners, between students and teachers.

\section{4 support on information organizational form}

Associating features is to facilitate students to establish links between the old and new knowledge, making students to initiative obtain information, to stimulate students' self-exploration initiative and enthusiasm of knowledge. LC resources have diversity, in addition to text, graphics, images, audio and video resources, allowing students to hear his voice, and to reflect in its shape, making the objective things visualized and concreted, which will not only helps students to establish contact between the old and new knowledge content, but also improves learners' motivation and interest in learning, and improve the ability to apply knowledge ${ }^{[4]}$.

\section{LC-based deep learning mode}

\subsection{Basic Principles}

(1) follow the principle of promoting the overall development of students and improving students' scientific literacy

(2) follow the principle of improving the students' interest and promoting the comprehensive development of individual

(3) follow the unity principle of knowledge structure and cognitive structure

(4) follow the principle of building a "learning community"

\section{2 model design of depth study promoted by LC}

The core model design of depth study promoted by LC, is to promote interaction between the various elements to form a coordination mechanism to promote each other. The ideas of model design overall adhere to the principle of a consistent design, and coordinat between the various elements of design based on the theory of constructivist learning. Model design of depth study promoted by LC includes five key areas: target-design, resources-design, context-design, event-design, feedback-design, shown in Fig. 1.

LC, which supports learners in Deep learning process, plays a major role in the following: 


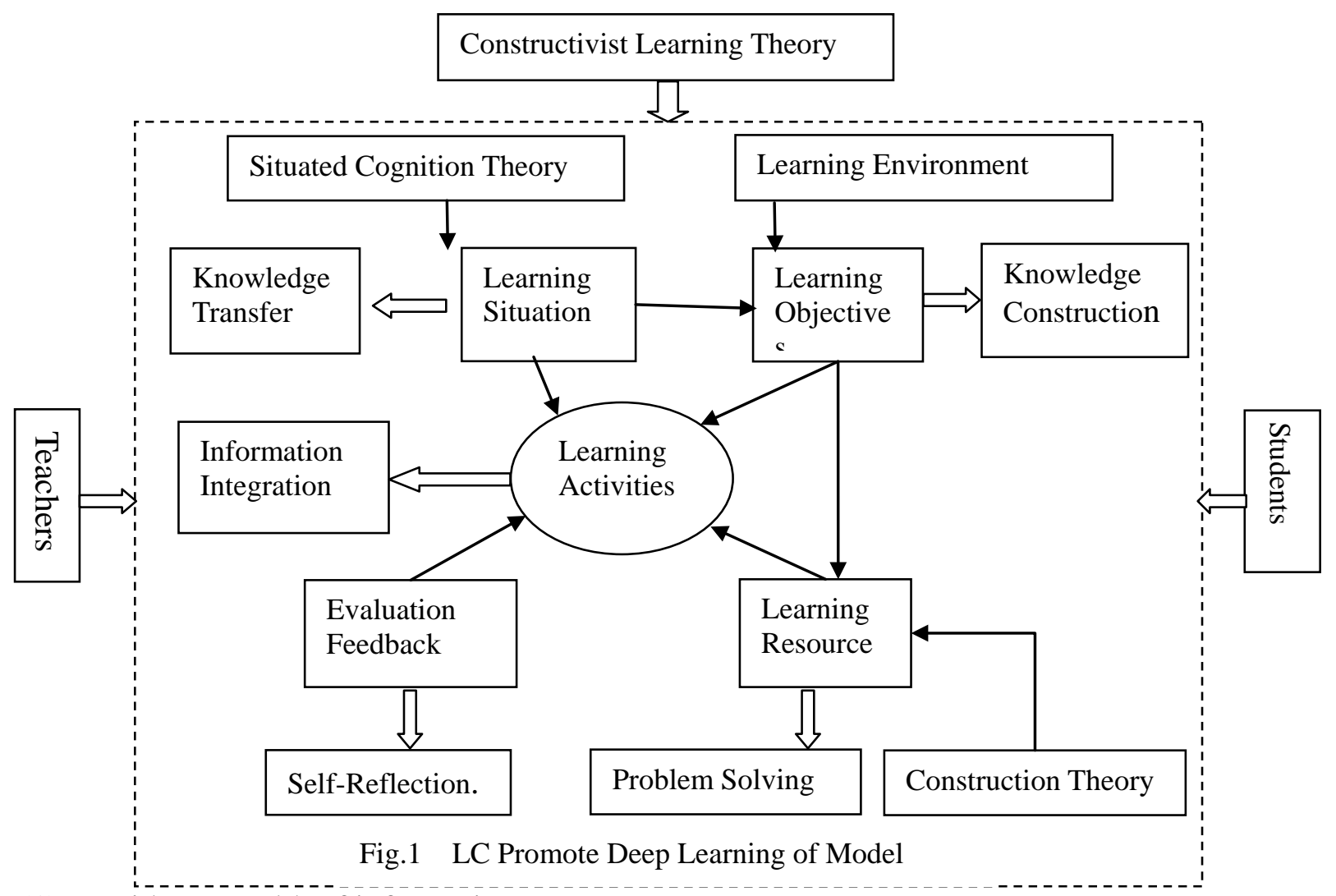

(1) provides a wealth of information resources

Deep learning needs of rich information resources as a learning content, the object of information processing. Depth learners must learn to clear the needs of information, to use search strategies, to storage and process the information, and to effective manage the available information resources.

(2) provides a rich interactive sharing intellectual resources

In the process of Deep learning, learner are required to continue to construct their own knowledge, skills, which means communication, interaction, negotiation. LC provides places such as network learning community, expert systems, virtual communities etc. for subject matter experts, community members, organizations personnel to fully communicate and interact, thus to effectively promote the construction and production of knowledge, skills.

(3) provides a tool of process management

LC provides a wealth tools of process management for the learners, which are self-awareness tools of learners, learning risk assessment tools, knowledge management tools, management tools, productivity tools which can improve learner performance, such as knowledge, translation software.

(4) provides evaluation tools

Evaluation of learning, some focus on the learning process, and some focus on learning outcomes, while deep learning focus on the learning process, reflecting that the learning process is more important than learning outcomes, therefore the Deep learning assessment tools are different from shallow learning.

Several issues should be noted in deep learning supported by LC

(1) Unicom Design

Unicom learning takes place in the initial stages of learning, that is learning the source of resources. The occurrence of depth study needs for clear learning objectives and abundant and alternative learning resources, so it is necessary to ensure the smooth flow of information channels.

(2) Interactive Design

Interaction is always, anywhere existed in learning, which is the interaction of people and resources, of people and people, and of people and environment. Learners interact with the physical environment is mainly operational use of the device. Learners immersed in the LC-depth study, are both producers and consumers of tacit knowledge, the words and deeds of each member have an 
impact on the specific content of tacit knowledge.

(3) Safety Design

Security design mainly involves three aspects, first is available sources of information, second is inspection, maintenance and updates equipment, third is learner equity. We must establish clear rules and regulations to raise the interests awareness of members, regulate the behavior of members, and effectively protect the interests of everyone.

\section{Conclusion}

Deep learning supported LC is a vitality learning, and take care of the students' learning concept, interpersonal skills, etc. on the basic of developing students 'higher-order thinking skills, knowledge of high-level skills. It reflects in the overall impact of education to human survival and development. The current wisdom education is loud voices, the construction step of wisdom classrooms is taking strides forward, LC can provide wisdom reference in classroom building constructive.

\section{Acknowledgements}

The paper is Supported by Natural Science Foundation of Hainan Province of China. (Grant No: 714255).

\section{Reference}

[1]. Zhang Ting: The integration of Learning Commons and Ubiquitous Learning Environments of the university library[D], Fujian Normal University, China,2013. p.25-27.

[2]. Zhang Hao, xiu-juan wu. The connotation of the deep learning and cognitive theory analysis[J].China Educational Technology,2012(10),p.7-11,21.

[3]. Chen Qi, Liu Rude educational psychology [M] Beijing: Higher Education Press, 2005: 138-156.

[4]. The theoretical basis of learning environments Jonathan [M] any of the Friends group, translated Shanghai:East China Normal University Press, 2002: 38-43.

[5]. Lynch Raymond; McNamara Patricia Mannix; Seery Niall. Promoting deep learning in a teacher education programme through self- and peer-assessment and feedback[J] European Journal of Teacher Education. 2012(2): 179-197.

[6]. DeLotell Pam Jones, Millam Loretta A, Reinhardt Michelle M. The Use Of Deep Learning trategies In Online Business Courses To Impact Student Retention [J]. Journal of Business\& Economics Research, 2010(12):49-55.

[7].M. Betul Yilmaz, Feza Orhan. The use of Internet by high school students for educational purposes in respect to their learning approaches[J]. Social and BehavioralSciences,2010(2):2143-2150. 